\title{
SATISFACCIÓN Y LEALTAD DEL VISITANTE EN ÁREAS PROTEGIDAS DE LA REPÚBLICA DOMINICANA. EL CASO DEL MONUMENTO NATURAL SALTOS DE LA DAMAJAGUA
}

Satisfaction and loyalty of the visitor in protected areas of the República Dominicana. the case of the natural monument saltos de la damajagua

\author{
Dr. FRANCISCO ORGAZ AGÜERA ${ }^{1}$ \\ Vicerrector de Producción e Investigación Científica de la Universidad Tecnológica de \\ Santiago \\ Dr. SALVADOR MORAL CUADRA ${ }^{2}$ \\ Profesor-Investigador de la Universidad Tecnológica de Santiago
}

\begin{abstract}
Resumen: El ecoturismo se conforma como una actividad turística la cual tiene en cuenta el medio donde es desarrollada dicha actividad, obteniéndose beneficios para la población local donde se inserta y llevada a cabo desde una perspectiva sostenible. El objetivo de este trabajo es el de realizar un análisis de las valoraciones de la lealtad y la satisfacción de los ecoturistas que visitan el Monumento Natural Saltos de la Damajagua, efectuando una metodología cuantitativa basada en la realización de encuestas a estos ecoturistas, donde la satisfacción, y por ende, la lealtad, queda demostrada, volviendo a visitar el destino, o bien, recomendándolo a familiares y/o amigos.
\end{abstract}

Palabras clave: Satisfacción, lealtad, área protegida, ecoturismo, República Dominicana

\begin{abstract}
Ecotourism is formed as a tourist activity which takes into account the environment where this activity is developed, obtaining benefits for the local population where it is inserted and carried out from a sustainable perspective. The objective of this work is to perform an analysis of the loyalty and satisfaction ratings of ecotourists visiting the Natural Monument of Saltos de la Damajagua, using a quantitative methodology based on conducting surveys of these ecotourists, where satisfaction, and therefore, the loyalty, is demonstrated, revisiting the destination, or, recommending it to relatives and / or friends.
\end{abstract}

Keywords: Satisfaction, loyalty, protected area, ecotourism, Dominican Republic.

\section{INTRODUCCIÓN}

Actualmente no existe un consenso sobre el origen del término "ecoturismo". Weaver y Lawton (2007) citan a Budowski (1976) como la primera persona que reconoce que la relación entre turismo y medio ambiente tiende a ser conflictiva, pero que existen potencialidades para una relación simbiótica basada en beneficios mutuos. Aunque, se ha llegado a imponer el término acuñado por Ceballos-Lascurain (1987), refiriéndose a ecoturismo como una forma de turismo que consiste en visitar áreas naturales relativamente

\footnotetext{
${ }^{1}$ Correo electrónico: franorgaz@utesa.edu

${ }^{2}$ Correo electrónico: salvamoral@utesa.edu
} 
intactas o poco alteradas, con el objeto de estudiar y admirar el paisaje, la flora y los animales salvajes que acogen, así como toda manifestación cultural (pasada o presente) observable en estas zonas.

La Unión Internacional para la Conservación de la Naturaleza (UICN) ha adoptado una nueva versión del concepto de ecoturismo realizada por Ceballos-Lascurain (1996), refiriéndose a aquella modalidad turística ambientalmente responsable consistente en viajar o visitar áreas naturales relativamente sin disturbar, con el fin de disfrutar, apreciar y estudiar los atractivos naturales (paisaje, flora y fauna silvestres) de dichas áreas, así como cualquier manifestación cultural (del presente y del pasado) que puedan encontrarse ahí, a través de un proceso que promueve la conservación, tiene bajo impacto ambiental y cultural y propicia un involucramiento activo y socio-económicamente benéfico de las poblaciones locales. En definitiva, el ecoturismo es una tipología turística que promueve actividades de turismo en la naturaleza, la conservación de la naturaleza y la generación de beneficios en las comunidades locales (Lu y Stepchenkova, 2012).

La Organización Mundial del Turismo no ha definido el término de forma oficial (OMT, 2002), si bien, ha desarrollado una serie de características generales para el término ecoturismo (Puertas Cañaveral, 2007): se trata de un turismo basado en la naturaleza y cuya motivación principal es la observación de la misma por parte del turista, al igual que el disfrute de la cultura dominante en las áreas naturales; está compuesto por elementos de interpretación y de educación; el viaje suele estar organizado por empresas especializadas, pequeñas y de propiedad local, y se dirigen a grupos minoritarios; intenta no producir impactos negativos en el medio natural ni en la sociedad y cultura dominante en las áreas naturales; mejora la protección de las áreas protegidas, debido a que genera recursos económicos para la población local, las empresas locales y la administración anfitriona; ofrece oportunidades de empleo a la población local; $\mathrm{y}$, crea concienciación y sensibilización de la población local y los turistas hacía la conservación de los recursos naturales y culturales.

Entre los componentes que fomentan el desarrollo del ecoturismo, podemos citar la demanda ecoturística o ecoturistas, las actividades ecoturísticas en zonas naturales, los destinos o lugares ecoturísticos y zonas naturales protegidas, las infraestructuras ecoturísticas, los guías de ecoturismo y, por último, los stakeholders o agentes sociales relacionados con el desarrollo de la actividad del ecoturismo (Fennel y Eagle, 1990; Ceballos-Lascuraín, 1996; Pérez de las Heras, 1999; Wearing y Neil, 2000; Puertas Cañaveral, 2007).

En este sentido, este trabajo de investigación pretende analizar el ecoturismo en áreas protegidas de República Dominicana, el cual está experimentando en los últimos años un crecimiento, no todo lo fuerte que se desearía por los agentes privados, comunidad local e instituciones competentes en turismo, pero se está conformando como una oferta complementaria al principal tipo de turismo dominante en el país: el turismo de sol y playa (Castellanos Verdugo y Orgaz Agüera, 2013). De esta manera, el objetivo de esta investigación es analizar las valoraciones sobre satisfacción y lealtad percibidas por los ecoturistas que visitan el Monumento Natural Saltos de la Damajagua, el cuál se ha consolidado como uno de los principales recursos turísticos naturales del país.

Este artículo se estructura, tras esta breve introducción, en un segundo apartado donde se muestra el marco teórico del estudio. En un tercer apartado, se describe la metodología de la investigación empleada en este trabajo. Posteriormente, se presentan los resultados del estudio. En un quinto apartado, se desarrollan las conclusiones, además de las implicaciones 
para la gestión, las limitaciones y las futuras líneas de investigación. Por último, se enumeran, en orden alfabético, las referencias bibliográficas citadas en el cuerpo del artículo.

\section{MARCO TEÓRICO}

En el ámbito turístico, entendemos la satisfacción como el estado mental del turista tras la vivencia de una experiencia determinada (Williams y Soutar, 2009), siendo función entre las expectativas que se producen previas a un viaje y las experiencias vividas tras dicho viaje (Chen y Chen, 2010).

La satisfacción de los turistas es un elemento a considerar para mejorar la gestión y promoción de un área turística, y por esta razón existen diversos destinos y organizaciones que desarrollan encuestas de satisfacción dirigidas a los visitantes (Zabkar et al., 2010). Así, mientras más competitivo sea un destino, más alta será la satisfacción de los visitantes (Mazanec et al., 2007), lo que conlleva a incrementar el número de turistas y de ingresos en el lugar. El grado de satisfacción de los consumidores con un destino turístico determinado depende de las expectativas del visitante con respecto a los atributos del destino y a la medida en que se cumplen esas expectativas (Clemons y Woodruff, 1992). Por tanto, es fundamental que los gestores turísticos reconozcan que las experiencias de los consumidores sobre las actividades que se realizan durante la estancia en un destino vacacional es probable que sean un factor determinante para la satisfacción de estos (Martín-Ruíz et al., 2010).

Siguiendo a Erevelles y Leavitt (1992), el cliente crea sus expectativas sobre el producto en la etapa anterior a la compra, ejerciendo un juicio tras la experiencia del consumo, con la comparación entre el resultado y sus expectativas a priori. De esta forma, la satisfacción del turista en el ecoturismo depende de las experiencias, proporcionándose una experiencia ecoturística satisfactoria cuando existe un comportamiento responsable hacia el medio ambiente (Lee y Moscardo, 2005). La satisfacción del cliente es lo que actuará como base para formar las actitudes e intenciones posteriores (Parasuraman, 1981). Según MartínRuíz et al. (2010), los clientes con los niveles de satisfacción más altos tendrán intenciones de uso más elevados que los clientes con los niveles de satisfacción más bajos.

Algunos autores (Court y Lupton, 1997; Bigné et al., 2001) han demostrado que la imagen de un destino afecta positivamente tanto a la intención de regresar de nuevo en el futuro como a la intención de recomendarlo. Así, la lealtad del consumidor se manifiesta en una variedad de comportamientos, siendo los más comunes la repetición de la compra o visita y la intención de recomendar la compra o visita (Konecnik y Gartner, 2007; Chi y Qu, 2008; Boo et al., 2009; Pike et al., 2010; Sirdeshmukh et al., 2002), siendo una de las variables que más han sido abordadas y estudiadas relativas a los comportamientos del consumidor (Moliner Velázquez et al., 2011). La evaluación de los consumidores y las intenciones del comportamiento han sido confirmados como dos elementos con relación positiva en la actividad turística (Lee et al., 2005). Así, las evaluaciones positivas aumentan la probabilidad de que el consumidor recomiende el servicio turístico a los demás (Martín-Ruiz et al., 2010). De esta forma, Martín-Ruiz et al. (2010) concluyeron que los visitantes eran mucho más propensos a recomendar una visita a otras personas que a repetir la visita por ellos mismos. La conceptualización de la lealtad ha sido comprendida como la prioridad para visitar o recomendar un determinado destino (Faullant et al., 2008; Li y Petrick, 2008; Prayag y Ryan, 2012). 
De esta manera, la demanda leal podría recomendar el destino a sus amigos y familiares y, por lo tanto, actuar en calidad de agentes publicitarios a través del boca-oreja (Murias Fernández et al., 2013). Según Gartner (1994), las recomendaciones de los visitantes previos son consideradas la fuente más fiable de información para los turistas potenciales. La lealtad se considera como una de las mayores fuentes de ventaja competitiva para un destino (Bharadwaj et al., 1993). De esta manera, los niveles de lealtad elevados fomentan la consolidación del destino en el mercado (Suárez Álvarez et al., 2007). Cabe destacar que los turistas que repiten visita gastan más que los que acuden por primera vez, aunque, los resultados de los estudios aplicados no son suficientemente concluyentes (Opperman, 2000).

\section{METODOLOGÍA DE LA INVESTIGACIÓN}

Tras realizar varias entrevistas a profesionales expertos en ecoturismo de la República Dominicana, se optó por desarrollar el estudio empírico en el área protegida Monumento Natural Saltos de la Damajagua (figura 1). Las razones principales fueron las siguientes:

- La posición estratégica, debido a que tal área protegida recibe una importante cantidad de personas extranjeras y nacionales de todo el país.

- $\quad$ Es el área protegida con mayor crecimiento de visitas los últimos años.

- $\quad$ La provincia de Puerto Plata, ubicada al norte del país, pasó de ser el principal polo turístico de la República Dominicana en los años 90, a ser el tercero (segundo de sol y playa). Actualmente, se están desarrollando estrategias turísticas encaminadas a mejorar las actividades complementarias y la diversificación de la oferta, lo que está haciendo que vuelva a crecer. La visita al Monumento Natural Saltos de la Damajagua forma parte de estas nuevas estrategias.

- $\quad$ El Monumento Natural Saltos de la Damajagua está considerado por los expertos del Ministerio de Medio Ambiente y Recursos Naturales de la República Dominicana como un "ejemplo a seguir" en materia de recursos naturales y turísticos co-manejados por los diferentes stakeholders del destino, por los importantes beneficios que genera para las comunidades locales.

El Monumento Natural Saltos de la Damajagua forma parte del Sistema Nacional de Áreas Protegidas de República Dominicana desde el 30 de junio de 2004. La extensión de esta área natural es de $6 \mathrm{~km}^{2}$ (600 hectáreas), albergando la cuenca del río que lleva su mismo nombre. Su localización exacta está en el municipio de Imbert (Puerto Plata), aunque comparte una porción con el municipio de Altamira (Puerto Plata), al norte de República Dominicana. Su acceso se encuentra en la carretera que une Santiago de los Caballeros con Puerto Plata, en concreto, en el tramo Llanos de Pérez. Esta área protegida tiene una peculiaridad en cuanto a su manejo, debido a que es un recurso que se administra bajo la modalidad de co-manejo, entre diferentes organizaciones e instituciones: Ministerio de Medio Ambiente y Recursos Naturales, Asociación de Guías del Río Damajagua, Ministerio de Turismo, Gobernación provincial Puerto Plata, Ayuntamiento de Imbert, Asociación de Hoteles, condominios y establecimientos comerciales de Playa Dorada, la Administración del Monumento Natural, y los propietarios del terreno del Monumento Natural (Orgaz Agüera y Moral Cuadra, 2014).

Los principales atractivos de esta zona natural son el río Damajagua, donde se realiza la actividad conocida como "barranquismo" o caminata por el curso del río, sorteando las 
diferentes dificultades que ofrece el recorrido. En todo momento, el visitante realiza el recorrido (llamado 27 Charcos) en contacto con diferentes especies de flora y fauna. El visitante tiene tres opciones: Realizar las excursiones de 27 charcos, de 12 charcos o de 7 , cada una con un precio diferente, y la contratación de uno de los recorridos incluye equipos y guías. Además de la actividad, se ofertan varios servicios más, como restaurante buffet, bar, tiendas con productos artesanales, fotografías y vídeos, y zapatos para la actividad. Además, el lugar está dotado con un Centro de Visitantes, baños, senderos y parking para los vehículos (Orgaz Agüera y Moral Cuadra, 2014).

\section{Figuras 1. Localización del Monumento Natural Saltos de Damajagua en la República Dominicana.}

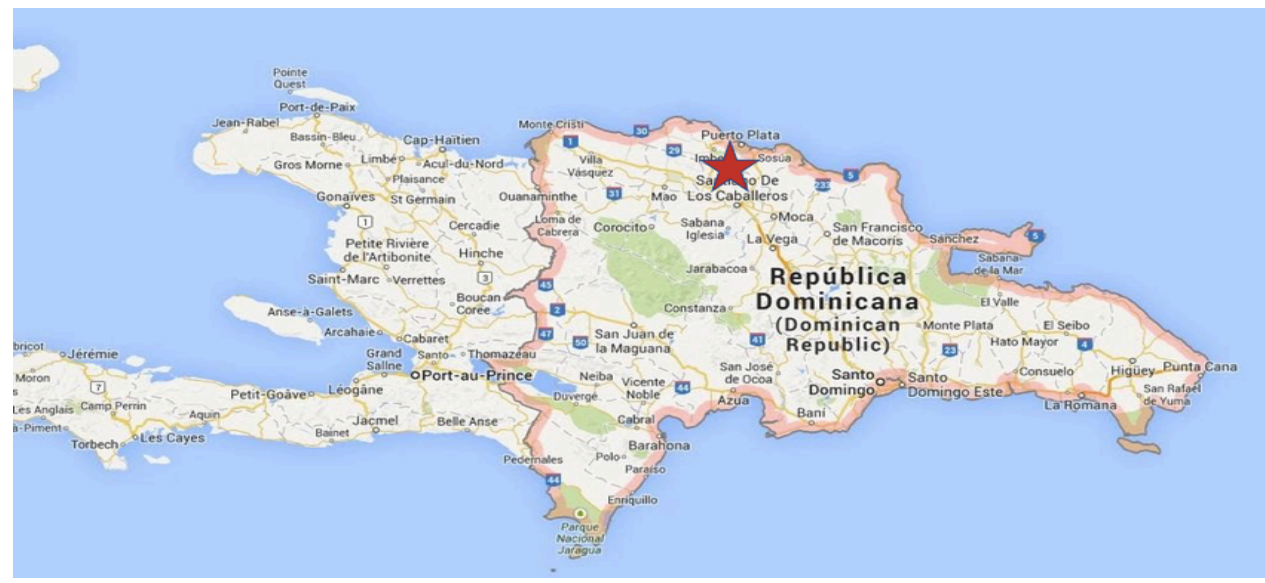

Fuente: elaboración propia.

La población objeto de estudio de este trabajo está compuesta por los ecoturistas que visitan el Monumento Natural Saltos de la Damajagua. El método elegido para la recogida de los datos ha sido la entrevista personal apoyada en un cuestionario estructurado. Con el fin de garantizar la validez del cuestionario, la formulación de los ítems se basó en ítems seleccionados de investigaciones previas (Yoon et al., 2010; Zabkar et al., 2010; Martín-Ruiz et al., 2010; Zhang y Lei, 2012). A partir de este conjunto inicial de ítems, se siguió un proceso de depuración de escalas en dos fases. Primero, un investigador especializado en turismo analizó los ítems propuestos; segundo, el cuestionario resultante fue revisado por un responsable del recurso turístico. De esta forma, se comprobó dos veces la validez de los ítems.

El instrumento de medida diseñado está estructurado en dos secciones. La primera sección examina las valoraciones de los visitantes referentes a la satisfacción y lealtad hacia el destino ecoturístico, mediante una escala de Likert de 5 puntos, donde 1 es igual a "muy en desacuerdo" y 5 hace referencia a "muy de acuerdo", siendo 3 un valor que indica indiferencia. Esta sección está formada por 12 ítems. La segunda sección trata sobre los aspectos sociodemográficos de los visitantes. De esta manera, se estudia el sexo, estado civil, nacionalidad, edad, actividad laboral, acompañantes y veces al año que practica ecoturismo. El alfa de Cronbach de los ítems del cuestionario es 0,829 , el cual se considera aceptable puesto que está por encima de 0,7 (Nunnally y Berstein, 1994).

El trabajo de campo se desarrolló sin incidentes en las tres últimas semanas del mes de febrero de 2014, logrando obtener un total de 520 cuestionarios válidos. Una vez cerrado el proceso de recepción de los cuestionarios se procedió a la tabulación de los datos en el programa Excel, para su posterior análisis en el programa estadístico IBM SPSS 24. 


\section{RESULTADOS DE LA INVESTIGACIÓN}

En relación al perfil de los visitantes, la muestra está compuesta en un $50 \%$ por mujeres y en la misma cantidad por hombres, siendo un $61,7 \%$ soltero/a y un $65,4 \%$ de nacionalidad no dominicana o extranjera (destacando Estados Unidos con el 34,2\%, solo superado por el $34,6 \%$ que corresponde a la cantidad de visitantes dominicanos residentes en el país). Referente a la edad, un 67,5\% de los visitantes tienen entre 18 y 34 años, con lo cual se puede deducir que los principales grupos de visitantes son relativamente jóvenes. Por su parte, un $39,8 \%$ son trabajadores por cuenta ajena y el 38,5\% hacen una vez ecoturismo al año. En la tabla 1 se muestran los principales datos relacionados al perfil de los turistas que visitan el Monumento Natural Saltos de la Damajagua.

Tabla 1. Perfil de los visitantes.

\begin{tabular}{|c|c|c|c|}
\hline Variable & $\%$ & Variable & $\%$ \\
\hline Sexo & & Estado civil & \\
\hline Masculino & $50,0 \%$ & Casado & $38,3 \%$ \\
\hline Femenino & $50,0 \%$ & Soltero & $61,7 \%$ \\
\hline País del visitante & & Edad & \\
\hline República Dominicana & $34,6 \%$ & $18-25$ & $31,2 \%$ \\
\hline Estados Unidos & $34,2 \%$ & $26-34$ & $36,3 \%$ \\
\hline Canadá & $9,0 \%$ & $35-44$ & $21,7 \%$ \\
\hline Francia & $3,8 \%$ & $45-54$ & $7,9 \%$ \\
\hline Alemania & $3,3 \%$ & $55-64$ & $1,9 \%$ \\
\hline Rusia & $3,1 \%$ & 65 o más & $1,0 \%$ \\
\hline Otros & $12,0 \%$ & & \\
\hline Actividad laboral & & Veces que hace ecoturismo/año & \\
\hline Estudiante & $30,6 \%$ & $1 \mathrm{vez}$ & $38,5 \%$ \\
\hline Trabajador por cuenta propia & $20,2 \%$ & 2 veces & $30,2 \%$ \\
\hline Trabajador por cuenta ajena & $39,8 \%$ & 3 veces & $10,4 \%$ \\
\hline Desempleado & $1,7 \%$ & Más de 3 veces & $21,0 \%$ \\
\hline Jubilado & $3,5 \%$ & & \\
\hline Labores del hogar & $4,2 \%$ & & \\
\hline
\end{tabular}

Fuente: elaboración propia.

En relación a las valoraciones con la satisfacción, estas han sido altas. En consonancia con los resultados obtenidos en la tabla 2, se puede afirmar que los valores medios de los ítems relacionados con la satisfacción estuvieron comprendidos entre 4 y 5 , lo que nos indica que, en general, los participantes de esta investigación muestran un cierto grado de acuerdo con todos los ítems planteados. La satisfacción global media es de 4,781 puntos sobre $5 \mathrm{y}$, por tanto, la satisfacción de los ecoturistas es alta. 
Tabla 2. Valoraciones sobre satisfacción.

\begin{tabular}{|c|c|c|c|c|c|c|}
\hline Ítem & $\mathbf{1}$ & $\mathbf{2}$ & $\mathbf{3}$ & $\mathbf{4}$ & $\mathbf{5}$ & Media \\
\hline $\begin{array}{c}\text { Estoy encantado de haber decidido } \\
\text { visitar esta área protegida }\end{array}$ & $0,4 \%$ & $0,0 \%$ & $1,0 \%$ & $15 \%$ & $83,7 \%$ & 4,815 \\
\hline $\begin{array}{c}\text { Estoy encantado de haber visitado } \\
\text { esta área protegida }\end{array}$ & $0,4 \%$ & $0,2 \%$ & $1,0 \%$ & $15,8 \%$ & $82,7 \%$ & 4,802 \\
\hline $\begin{array}{c}\text { Esta visita ha superado mis } \\
\text { expectativas }\end{array}$ & $0,4 \%$ & $0,4 \%$ & $3,3 \%$ & $17,7 \%$ & $78,3 \%$ & 4,731 \\
\hline $\begin{array}{c}\text { Me encuentro contento por decidir } \\
\text { venir a esta área protegida }\end{array}$ & $0,2 \%$ & $0,0 \%$ & $1,3 \%$ & $17,3 \%$ & $81,2 \%$ & 4,792 \\
\hline $\begin{array}{c}\text { En general, estoy satisfecho con } \\
\text { esta visita }\end{array}$ & $0,6 \%$ & $0,0 \%$ & $1,3 \%$ & $17,9 \%$ & $80,2 \%$ & 4,771 \\
\hline $\begin{array}{c}\text { En general, estoy contento con esta } \\
\text { visita }\end{array}$ & $0,2 \%$ & $0,0 \%$ & $1,5 \%$ & $18,1 \%$ & $80,2 \%$ & 4,781 \\
\hline $\begin{array}{c}\text { Creo que hice lo correcto en elegir } \\
\text { visitar esta área protegida }\end{array}$ & $0,6 \%$ & $0,2 \%$ & $0,8 \%$ & $17,7 \%$ & $80,8 \%$ & 4,779 \\
\hline \multicolumn{7}{|c|}{ Satisfacción global media: 4,781 sobre 5 } \\
\hline
\end{tabular}

Fuente: elaboración propia.

En la tabla 3 se muestran los resultados de las valoraciones referentes a la lealtad de los visitantes. En consonancia con los resultados obtenidos en dicha tabla, se puede afirmar que los valores medios de los ítems relacionados con la lealtad estuvieron comprendidos entre 4 y 5, lo que nos indica que, en general, los participantes de esta investigación muestran un cierto grado de acuerdo con todos los ítems planteados. La lealtad global media es de 4,768 puntos sobre 5, lo que nos confirma el alto grado de lealtad de los visitantes respecto al Monumento Natural Saltos de la Damajagua.

Tabla 3. Valoraciones sobre lealtad.

\begin{tabular}{|c|c|c|c|c|c|c|}
\hline Ítem & $\mathbf{1}$ & $\mathbf{2}$ & $\mathbf{3}$ & $\mathbf{4}$ & $\mathbf{5}$ & Media \\
\hline $\begin{array}{c}\text { Es probable que recomiende esta } \\
\text { área protegida a familiares y amigos }\end{array}$ & $0,2 \%$ & $0,0 \%$ & $1,2 \%$ & $18,5 \%$ & $80,2 \%$ & 4,785 \\
\hline $\begin{array}{c}\text { En otra ocasión me gustaría repetir } \\
\text { la visita a esta área protegida }\end{array}$ & $0,4 \%$ & $1,0 \%$ & $1,7 \%$ & $18,8 \%$ & $78,1 \%$ & 4,733 \\
\hline $\begin{array}{c}\text { Si tengo que decidir de nuevo, } \\
\text { elegiría esta área protegida } \\
\text { nuevamente }\end{array}$ & $0,8 \%$ & $0,2 \%$ & $1,9 \%$ & $19,6 \%$ & $77,5 \%$ & 4,729 \\
\hline $\begin{array}{c}\text { Les recomendaré esta práctica } \\
\text { ecoturística a amigos y familiares }\end{array}$ & $0,2 \%$ & $0,0 \%$ & $0,8 \%$ & $17,5 \%$ & $81,5 \%$ & 4,802 \\
\hline $\begin{array}{c}\text { Les hablaré muy bien de esta área } \\
\text { protegida a amigos y familiares }\end{array}$ & $0,2 \%$ & $0,2 \%$ & $0,6 \%$ & $17,5 \%$ & $81,5 \%$ & 4,800 \\
\hline \multicolumn{7}{|c|}{ Lealtad global media: 4,768 sobre 5 } \\
\hline
\end{tabular}


Fuente: elaboración propia.

El valor medio de las respuestas a los ítems referidos a la satisfacción y lealtad señala que los encuestados perciben de forma satisfactoria su visita al Monumento Natural Saltos de la Damajagua.

\section{CONCLUSIONES, IMPLICACIONES PARA LA GESTIÓN, LIMITACIONES Y FUTURAS LÍNEAS DE INVESTIGACIÓN}

La revisión de la literatura realizada en esta investigación ha puesto de manifiesto que existen un gran número de estudios sobre ecoturismo (Weaver y Lawton, 2007), analizándose en ellos una gran diversidad de temas como la percepción de los residentes, motivaciones de los turistas, potencialidades de un destino, identificación de recursos ecoturísticos, actitudes hacia el ecoturismo, implicaciones del ecoturismo y revisiones de literatura científica, entre otros. Por otro lado, esta revisión de la literatura también muestra los escasos estudios sobre turismo en la República Dominicana y, en consecuencia, aspectos como las motivaciones y valoraciones de los turistas, la legislación turística o las potencialidades de nuevos tipos de turismo no han recibido la debida atención por parte de los investigadores.

Referente al ecoturismo, se ha observado que, entre el gran número de investigaciones existentes, el concepto de ecoturismo ha sido definido por numerosos autores e investigadores, llegando Fennell (2001) a identificar más de 85 definiciones de este concepto hasta el año 2001. Entre todas las definiciones de ecoturismo, una de las definiciones más completas, bajo nuestro punto de vista, es la de Lu y Stepchenkova (2012), quien define el ecoturismo como una tipología turística que promueve actividades de turismo en la naturaleza, la conservación de la misma y la generación de beneficios en las comunidades locales.

En relación al análisis de los ecoturísticas que visitan el Monumento Natural Saltos de la Damajagua, esta investigación ha puesto de manifiesto que los principales ecoturistas que visitan el recurso son de origen extranjero. Así, también se ha podido constatar que los ecoturistas perciben de forma satisfactoria su visita al recurso. En este sentido, los visitantes quedan satisfechos y dispuestos a recomendar y repetir el viaje en esta área protegida. De esta manera, la satisfacción puede influir positivamente en la lealtad de los ecoturistas, ya sea a través de una nueva visita al recurso o recomendando el mismo a la demanda potencial, considerándose esto, y según Bharadwaj et al., (1993), como una de las mayores fuentes de ventaja competitiva para un destino. Estos datos no son insignificantes, dada la importancia que tiene la satisfacción de los turistas en la práctica del turismo, siendo esta crucial para la supervivencia y el éxito de un destino turístico, sobre todo, teniendo en cuenta la gran competencia que aparece a nivel internacional en este sector.

Los resultados de esta investigación pueden ser útiles, en primer lugar, para la Administración del Monumento Natural Saltos de la Damajagua, que está compuesta por varias organizaciones en co-manejo (Ministerio de Medio Ambiente y Recursos Naturales; Asociación de Guías del Río Damajagua; Ministerio de Turismo; Gobernación provincial Puerto Plata; Ayuntamiento de Imbert; Asociación de Hoteles, condominios y establecimientos comerciales de Playa Dorada; la Administración del Monumento Natural; y, los propietarios del terreno del Monumento Natural). Los gestores de este recurso deben seguir apostando por el desarrollo sostenible del mismo, puesto que genera numerosos empleos para la comunidad local, al igual que mejora la conservación del recurso natural. Así, 
apostar por la responsabilidad social corporativa debe seguir siendo el camino de mejorar la educación de los empleados y de las comunidades locales, debido a que un alto valor y calidad percibida mejorará la satisfacción de los visitantes. En este aspecto, los guías como principal recurso humano del recurso, deben mejorar su conocimiento de idiomas, sobre todo, y viendo la demanda que visita el recurso, el ruso y el alemán. Estas estrategias podrían fomentar que la satisfacción y lealtad del visitante siga siendo alta.

Entre las limitaciones de este estudio, los autores son conscientes de que un estudio longitudinal realizado en varios momentos temporales hubiese sido, sin lugar a duda, mucho más apropiado y atractivo, pero la insuficiencia de recursos de índole temporal, económico y humano, ha llevado a inclinarnos por una investigación de carácter transversal. También, el estudio está planteado únicamente desde el punto de vista la demanda ecoturística, dificultando la difusión de los descubrimientos a otros grupos de stakeholders relevantes para el turismo, como, por ejemplo, los empleados, los residentes o los administradores del recurso.

Como futuras líneas de investigación, sería interesante plantear estudios donde se analizará las diferencias en las percepciones, valoraciones y actitudes para diversos segmentos de la demanda del recurso. Por ejemplo, conocer si hay diferencias significativas entre las percepciones de la demanda ecoturística, población local, empleados o administradores locales. También se podría analizar si hay diferencias entre la demanda nacional y extranjeros, e incluso por género y edades.

\section{BIBLIOGRAFÍA}

BHARADWAJ, S. G., VARADARAJAN, P. R., y FAHY, J. Sustainable competitive advantage in service industries: a conceptual model and research propositions. En: The Journal of Marketing. 1993, pp. 83-99.

BIGNE, J. E., SANCHEZ, M. I., y SANCHEZ, J. Tourism image, evaluation variables and after purchase behaviour: inter-relationship. En: Tourism management. 2001, vol. 22, $n^{o}$ 6, pp. 607-616.

BOO, S., BUSSER, J., y BALOGLU, S. A model of customer-based brand equity and its application to multiple destinations. En: Tourism Management. 2009, vol. 30, no 2, pp. 219-231.

BUDOWSKI, G. Tourism and environmental conservation: conflict, coexistence, or symbiosis?" En: Environmental conservation. 1976, vol. 3, nº 1, pp. 27-31.

CASTELLANOS VERGUDO, M. y ORGAZ AGÜERA, F. Potencialidades ecoturísticas de la República Dominicana. En: TURyDES. 2013, vol. 6, nº 14, pp. 1-9.

CEBAllos-LASCURAIN, H. Estudio de Perfectibilidad Socioeconómica del Turismo Ecológico y Anteproyecto arquitectónico y urbanístico del Centro de Turismo Ecológico de Slan Kalan, Quintana Roo, México. México: SEDUE. 1987.

CEBALlOS-LASCURAIN, H. Tourism, Ecotourism and Protected Areas. Gland, Switzerland and Cambridge, UK: IUCN. 1996.

CHEN, C.-F. y CHEN, F.-S. Experience quality, perceived value, satisfaction and behavioral intentions for heritage tourists. En: Tourism Management. 2010, vol. 31, $\mathrm{n}^{\mathrm{o}}$ 1, pp. 2935 .

CHI, C.G.-Q. y QU, H. Examining the structural relationships of a destination image, tourist saisfaction and destination loyalty. An integrated approach. En: Tourism Management. 2008, vol. 29, nº 4, pp. 624-636. 
CLEMONS, D. S., y WOODRUFF, R. B. Broadening the view of consumer (dis) satisfaction: A proposed means-end disconfirmation model of CS/D. En: Marketing theory and applications. 1992, vol. 3, pp. 209-216.

COURT, B., y LUPTON, R. A. Customer portfolio development: Modeling destination adopters, inactives, and rejecters. En: Journal of Travel Research. 1997, vol. 36, $\mathrm{n}^{\mathrm{o}} 1$, pp. 35-43.

EREVELLES, S., y LEAVITT, C. A comparison of current models of consumer satisfaction/dissatisfaction. En: Journal of consumer satisfaction, dissatisfaction and complaining behavior. 1992, vol. 5, $\mathrm{n}^{\mathrm{o}}$ 10, pp. 104-114

FAULLANT, R., MATZLER, K., y FÜLLER, J. The impact of satisfaction and image on loyalty: The case of Alpine ski resorts. En: Managing Service Quality. 2008, vol. 18, $\mathrm{n}^{\mathrm{o}}$ 2, pp. 163-178.

FENNELL, D. A. A content analysis of ecotourism definitions. En: Current Issues in Tourism. 2001, vol. 4, no 5, pp. 403-421.

FENNELL, D. y EAGLES, P. Ecotourism in Costa Rica: A conceptual framework. En: Journal Park and Recreation Administration. 1990, vol. 1, nº 1, pp. 23-34.

GARTNER, W. C. Image formation process. En: Journal of travel \& tourism marketing. 1994, vol. 2, no 2-3, pp. 191-216.

KONECNIK, M., y GARTNER, W. C. Customer-based brand equity for a destination. En: Annals of Tourism Research. 2007, vol. 34, n² 2, pp. 400-421.

LEE, C. K., LEE, Y. K., y LEE, B. Korea's destination image formed by the 2002 World Cup. En: Annals of tourism research. 2005, vol. 32, $\mathrm{n}^{\circ} 4$, pp. 839-858.

LEE, W. H., y MOSCARDO, G. Understanding the impact of ecotourism resort experiences on tourists' environmental attitudes and behavioural intentions. En: Journal of sustainable tourism. 2005, vol. 13, $\mathrm{n}^{\mathrm{o}} 6$, pp. 546-565.

LI, X., y PETRICK, J. F. Examining the antecedents of brand loyalty from an investment model perspective. En: Journal of Travel Research. 2008, vol. 47, pp. 25-34.

LU, W. y STEPCHENKOVA, S. Ecotourism experiences reported online: Classification of satisfaction attributes. En: Tourism Management. 2012, vol. 33, no 3, pp. 702-712.

MARTÍN-RUIZ, D., CASTELLANOS-VERDUGO, M., y OVIEDO-GARCÍA, M. A. A visitors' evaluation index for a visit to an archaeological site. En: Tourism Management. 2010, vol. 31, no 5, pp. 590-596.

MAZANEC, J. A., WÖBER, K., y ZINS, A. H. Tourism destination competitiveness: from definition to explanation? En: Journal of Travel Research. 2007, vol. 46, n 1, pp. 8695.

MOLINER VELÁZQUEZ, B., GIL SAURA, I., y RUIZ MOLINA, M.E. Conceptualizing and measuring loyalty: towards a conceptual model of tourist loyalty antecedents. En: Journal of Vacation Marketing. 2011, vol. 17, $\mathrm{n}^{\mathrm{o}}$ 1, pp. 65-81.

MURIAS FERNÁNDEZ, P. M., GONZÁLEZ, D. R., y NOVELLO, S. Value added, authenticity and loyalty during the 2010 Xacobeo event: a tourists perception perspective. En: Cuadernos de Turismo. 2013, vol. 31, pp. 345-347.

NUNNALLY, J. y BERNSTEIN, I. Psychometric Theory. New York: McGraw-Hill. 1994.

OMT. Cumbre Mundial del Ecoturismo: informe final. Madrid: Organización Mundial del Turismo. 2002.

OPPERMANN, M. Tourism destination loyalty. En: Journal of travel research. 2000, vol. 39, $\mathrm{n}^{\mathrm{o}} 1$, pp. $78-84$. 
ORGAZ AGÜERA, F. y MORAL CUADRA, F. Las áreas protegidas como espacios para fomentar el desarrollo sostenible y el turismo comunitario. En: DELOS: Desarrollo Local Sostenible, 2014, no 20, pp. 1-8.

PARASURAMAN, A., LEONARD, B., y ZEITHAML, V. Measurement and evaluation of satisfaction process in retail setting. En: Journal of Retailing. 1981, vol. 57, pp. 25-48.

PÉREZ DE LAS HERAS, M. La guía del ecoturismo o cómo conservar la naturaleza a través del turismo. Madrid: Mundi Prensa. 1999.

PIKE, S., BIANCHI, C., KERR, G., y PATTI, C. Consumer-based brand equity for Australia as a long-haul tourism destination in an emerging market. En: International Marketing Review. 2010, vol. 27, pp. 434-449.

PRAYAG, G., y RYAN, C. Antecedents of tourists' loyalty to Mauritius: The role and influence of destination. En: Journal of Travel Research. 2010, vol. 51, no 3, pp. 342356.

PUERTAS CAÑAVERAL, I. Ecoturismo en las Reservas de la Biosfera. Granada: Universidad de Granada. 2007.

SIRDESHMUKH, D., SINGH, J., y SABOL, B. Consumer trust, value, and loyalty in relational exchanges. En: Journal of marketing. 2002, vol. 66, nº 1, pp. 15-37.

SUÁREZ ÁlVAREZ, L., CASIELLES, R. V., y MARTíN, A. M. D. La confianza y la satisfacción del cliente: variables clave en el sector turístico. En: Revista europea de dirección y economía de la empresa. 2007, vol. 16, $\mathrm{n}^{\mathrm{o}}$ 3, pp. 115-132.

WEARING, S. y NEIL, J. Ecoturismo: impacto, tendencias y posibilidades. Madrid: Sintesis. 2000.

WEAVER, D.B. y LAWTON, L.J. Twenty years on: The state of contemporary ecotourism research. En: Tourism Management. 2007, vol. 28, nº 5, pp. 1168-1179.

WILLIAMS, P., y SOUTAR, G.N. Value, satisfaction and behavioral intentions in an adventure tourism context. En: Annals of Tourism Research. 2009, vol. 36, $\mathrm{n}^{\mathrm{o}} 3$, pp. 413-438.

YOON, Y.S. y UYSAL, M. An examination of the effects of motivation and satisfaction on destination loyalty: a structural model. En: Tourism Management. 2005, vol. 26, pp. 4556.

ŽABKAR, V., BRENČIČ, M. M., y DMITROVIĆ, T. Modelling perceived quality, visitor satisfaction and behavioural intentions at the destination level. En:Tourism management. 2010, vol. 31, $\mathrm{n}^{\circ}$ 4, pp. 537-546.

ZHANG, H. y LEI, S.L. A structural model of residents' intention to participate in ecotourism: The case of a wetland community. En: Tourism Management. 2012, vol. $33, n^{\circ} 4$, pp. 916-925. 